\title{
人机安全交互技术研究进展及发展趋势*
}

\author{
贾计东 ${ }^{1,2}$ 张明路 $^{1}$ \\ (1. 河北工业大学机械工程学院 天津 300130; \\ 2. 机器人技术与系统国家重点实验室(哈尔滨工业大学) 哈尔滨 150006)
}

\begin{abstract}
摘要: 人机共融作为未来生活、智能工厂等方面焦点问题, 承载着新型工业体系构建以及衡量国家科技创新水平的重担。聚 焦人机安全交互关键技术, 围绕共融机器人结构设计、柔顺控制、环境感知、行为学习及决策等层面全面总结了当前研究进 展, 系统梳理了热点难点问题, 归纳了现阶段人机安全交互实现方法, 并对未来发展航向做出了展望。研究旨在通过抛砖引 玉，加深共性关键技术理解，促进相关学科领域全面发展。
\end{abstract}

关键词: 人机交互; 碰撞检测; 意图识别; 柔顺控制; 运动控制; 安全

中图分类号: TP242

\section{Research Progress and Development Trend of the Safety of Human-robot Interaction Technology}

\author{
JIA Jidong ${ }^{1,2}$ ZHANG Minglu ${ }^{1}$ \\ (1. School of Mechanical Engineering, Hebei University of Technology, Tianjin 300130; \\ 2. State Key Laboratory of Robotics and System (Harbin Institute of Technology), Harbin 150006)

\begin{abstract}
Human-Robot integration is the focus of future life, smart factories, and so on. It bears the burden of building a new industrial system and measuring the level of national scientific and technological innovation. Focusing on the key technologies of human-computer safety interaction, the current research progress is summarized around the structural design,compliance control,environment perception, behavioral learning and decision-making of the communicator systematically sorted out the hot and difficult issues. The realization of human-computer security interaction at this stage is summarized. The prospects for future development are pointed out. The purpose of this research is to promote the understanding of key technologies in common areas and promote the comprehensive development of related disciplines.
\end{abstract}

Key words: human-robot interaction; collision detection; intention recognition; compliance control; motion control; safety

\section{0 前言}

纵观机器人产业发展 50 多年, 其应用从工业制 造越来越多地渗透到家庭服务、医疗护理、航天科 技等诸多领域 ${ }^{[1-3]}$, 伴其而变的是工作环境逐渐由传 统结构单一化转为复杂多模态工作环境, 工作方式 也由最初的分离囚笼式向人机共融协同转化。随着 新一轮工业革命推进, 机器人技术作为先进智能制 造的载体，承担着改善人类生活方式以及衡量国家 科技创新水平的重任, 具有重要研究意义 ${ }^{[4-6]}$ 。而机

* 国家重点研发计划(2017YFB1302701)、机器人与系统国家重点实验室 (HIT)自行任务(SKLRS201816B)、河北省重点基金(E2018202338)和河北 工业大学联合培养(2017GN0008)资助项目。20190315 收到初稿, 20190730 收到修改稿
器人交互安全问题作为新一代共融机器人技术评价 基础, 将加强机器人与人以及非结构物理环境接触 的安全技术要求, 加剧机器人同人类生产生活安全 矛盾问题。

机器人的安全性作为机器人基本特征以及机器 人领域共性技术,一直受到广大学者和企业的青睐。 相比传统机器人安全要求, 新一代人机交互安全技 术更加强调人机共融。与人共融，顾名思义是让机 器人逐渐具备人类感知能力、学习能力、适应能力 以及决策能力, 结合人类大脑逻辑思维和应变能力, 充分发挥机器人快速、精准等机械性能, 形成机器 人与人优势互补。其安全性鲜明特点表征为机器人 与人共享相同自然空间、机器人与人自主交互、与 人双向协同、与人协调互补, 进而确保人机物安全, 拓展作业能力; 其共性技术特征在于基于人体危害 
感知的机器人安全行为评价准则, 基于传感器的环 境感知、意图识别技术, 以及基于人工智能的认知 学习、决断决策安全保障机制等。

根据机器人交互行为过程, 将人机安全交互问 题分为交互前危险预警和交互后安全保障, 即通常 意义下的碰撞避免、检测和响应等。本文主要围 绕人机交互流程中的安全问题, 以协作机器人、 医疗护理机器人等热点研究对象, 从机器人安全 评价准则、环境感知、行为学习及决断决策等共 性关键技术出发, 加以生物机电一体、意图感知 识别、人工智能等前沿技术, 分别从本体设计、 柔顺控制、行为预测及认知决策等层面对以往研 究进行总结、补充和拓展, 为具有安全交互性能 的新一代机器人设计、创新和发明提供系统的理 论基础和方法。

\section{1 人机交互安全问题描述}

人机交互技术涉及机构学、生物力学、机电一 体化、神经科学、计算机信息处理、先进控制理论 等多学科交叉融合。其主要分为物理人机交互 (Physical human-robot interaction, pHRI)和自然人机 交互(Social Human-Robot interaction, sHRI)。在交互 安全问题上, pHRI 强调人机物理接触, 着重引导 触觉感知安全; 而 SHRI 则主要包含以视觉图像处 理、语音识别等感官认知为特点的认知交互, 引 导识别系统算法可靠性安全问题。两种交互研究 侧重点不同, 互为个体; 但对于当前机器人高度 集成系统, 两种交互行为相辅相成。诸如接触后 感知与响应等行为以 $\mathrm{pHRI}$ 为输出表象, 执行控制 器由认知交互识别所生成环境信息命令; 而 sHRI 作为系统反馈输入, 采集物理交互行为结果, 给 控制器传递环境感知量, 多集中在预警与决策研 究, 如图 1 所示。

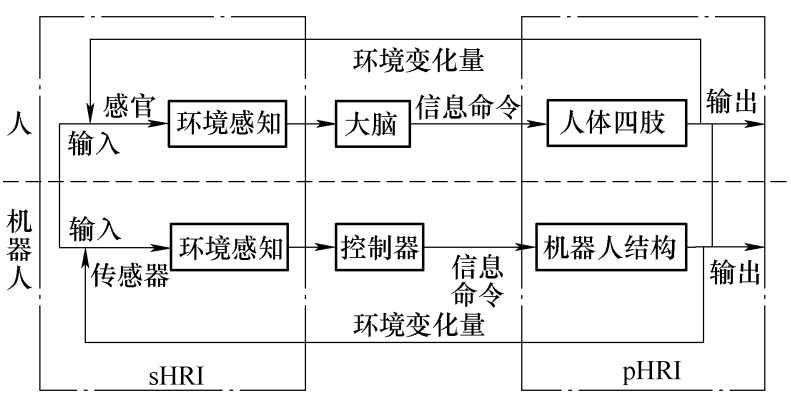

图 1 pHRI 和 sHRI 相互关系

根据应用载体不同, 机器人可分为面向智能制 造的协作机器人、面向家庭护理的医疗服务机器人、
面向智能仓储运输的移动机器人等等。然而, 无论 哪一类型机器人, 当前主要研究对象是面向共享空 间下 “以人为中心” 的安全共融机器人。当前研究 已实现了人与机器人共存以及简单交互问题, 如接 触前降速、停止, 接触后急停、阻抗或重力补偿响 应等; 然而机器人与人自主交互、与人双向协同、 协调互补等交互行为尚未形成统一框架。对于动态、 复杂的非结构环境感知、任务多样化以及自主交互 (包括多机器人平台与平台之间和平台与人之间交 互)等问题依然极富挑战。此外，拟人机械臂作为诸 多应用领域中代表性平台, 兼具传统机器人高效高 精度特性与仿人手臂灵活性, 逐渐被拓展为轮式、 足式双臂移动平台。如表 1 所示。其诸如驱动、传 感和控制等共性关键技术的创新设计与稳定性优 化, 依然成为当前研究热点, 旨在保证安全的同时 降低成本和提高工作效率, 为完成高效人机交互提 供技术支撑。

\section{2 机器人安全评价准则}

正如汽车行业安全技术评价标准, 机器人产 业同样应该拥有相关技术准则, 那么何以评价人 机交互技术安全等级? 根据科幻作家艾萨克·阿 西莫夫 ${ }^{[25]}$ 于 1942 年提出的三个 “机器人法则”, 其中第一条规定: “机器人不能伤害人类, 或者允 许人类受到伤害而坐视不理”。该条例作为三条规 则中的基础, 强调了机器人与人的本质从属关系 与安全要求。

机器人产业安全交互评价准则的发展与完 善, 得到诸多学者的研究贡献。研究初期, 为避 免人体伤害, 试验多基于模拟 ${ }^{[26]}$ 和实际物理硬件 平台 ${ }^{[27-28]}$, 集中于机器人与模拟假人进行碰撞测 试。随后 HADDADIN ${ }^{[29]}$ 将伤害知识嵌入控制系 统, 并从猪身上采集生物组织样本来模拟实际人 体组织与机器人间的碰撞危害试验, 用以提高机 器人安全感知能力; 同时针对真正的人与机器人 碰撞测试, POVSE 等 ${ }^{[30]}$ 将人体碰撞疼痛进行分 类, FRAUNHOFER IFF ${ }^{[31]}$ 详细研究了损伤阈值的 设定, HADDADIN ${ }^{[32]}$ 针对控制策略有效性展开进 一步调查说明, 相继在文献[33]中, HADDADIN 又详细讨论了当前标准的限制并提出改进意见。 此外, JUNG 等 ${ }^{[34]}$ 围绕人机共融环境下的服务机 器人伤害预防标准问题展开详细分析, 为个人护 理、医疗等相关安全技术评定的完善和改进提供 了依据。 
表 1 协作机械臂本体概述

\begin{tabular}{|c|c|c|c|c|c|}
\hline $\begin{array}{l}\text { 分 } \\
\text { 类 }\end{array}$ & $\begin{array}{l}\text { 文 } \\
\text { 献 }\end{array}$ & 名称 & $\begin{array}{l}\text { 研究 } \\
\text { 单位 }\end{array}$ & $\begin{array}{c}\text { 发布 } \\
\text { 时间/年 }\end{array}$ & 特征 \\
\hline \multirow{5}{*}{$\begin{array}{l}\text { 单 } \\
\text { 臂 }\end{array}$} & {$[7]$} & $\begin{array}{l}\text { UR3/5/ } \\
10\end{array}$ & $\begin{array}{l}\text { Universal } \\
\text { Robots }\end{array}$ & $\begin{array}{l}2015 \\
2008 \\
2010\end{array}$ & $\begin{array}{l}\text { UR 最早发布协作机器人, } 6 \text { 自由度, 关节采用双编码器, 结构简单, 负载分为 } 3 \mathrm{~kg} / 5 \mathrm{~kg} / 10 \\
\mathrm{~kg} \text { 系列, 重复定位精度可达 } \pm 0.1 \mathrm{~mm}\end{array}$ \\
\hline & {$[8]$} & LBR iiwa & KUKA & 2014 & $\begin{array}{l}\text { 空间 } 7 \text { 自由度, 关节采用扭矩传感器和双编码器, 直接力控制, 结构相对复杂, 负载 } 7 \mathrm{~kg} \text {, } \\
\text { 重复定位精度 } \pm 0.1 \mathrm{~mm}\end{array}$ \\
\hline & [9] & Sawyer & Rethink & 2015 & $\begin{array}{l}\text { 空间 } 7 \text { 自由度, 关节采用 SEA 弹性元件, 结构相对复杂, 负载 } 4 \mathrm{~kg} \text {, 自重 } 19 \mathrm{~kg} \text {, 重复定位 } \\
\text { 精度 } \pm 0.1 \mathrm{~mm}\end{array}$ \\
\hline & [10] & $\begin{array}{l}\text { Franka } \\
\text { Emika }\end{array}$ & Franka Emika & 2017 & $\begin{array}{l}\text { 空间 } 7 \text { 自由度, 关节采用应变式压力传感器, 负载 } 3 \mathrm{~kg} \text {, 重复定位精度 } \pm 0.1 \mathrm{~mm} \text {, 最大优 } \\
\text { 势是成本较低 }\end{array}$ \\
\hline & [11] & Gen3 & KINOVA & 2018 & 空间 6 自由度, 嵌入 $2 \mathrm{D} / 3 \mathrm{D}$ 视觉和快速连接控制器, 各关节装载力矩传感器, 负载 $2.6 \mathrm{~kg}$ \\
\hline \multirow{4}{*}{$\begin{array}{l}\text { 双 } \\
\text { 臂 }\end{array}$} & [12] & $\operatorname{Cog}$ & 麻省理工 & 2002 & 双臂 12 自由度, 关节装载 SEA 弹性元件, 内部附有视觉、力觉等传感器 \\
\hline & [13] & Sda 系列 & 安川 & 2012 & 双臂 14 自由度，仿人尺寸，具有较高灵活性、精准性和协调性 \\
\hline & [14] & Baxter & Rethink & 2014 & $\begin{array}{l}\text { 双臂具有 } 14 \text { 自由度, 关节采用 SEA 弹性元件, 具有强柔性特性, 负载 } 2.2 \mathrm{~kg} \text {, 自重 } 74 \mathrm{~kg} \text {, } \\
\text { 重复定位精度 } \pm 0.5 \mathrm{~mm}\end{array}$ \\
\hline & [15] & YuMi & $\mathrm{ABB}$ & 2014 & 双臂 14 自由度, 采用轻量化设计, 负载 $0.5 \mathrm{~kg}$, 自重 $38 \mathrm{~kg}$, 重复定位精度 $\pm 0.02 \mathrm{~mm}$ \\
\hline \multirow{5}{*}{$\begin{array}{l}\text { 轮 } \\
\text { 式 }\end{array}$} & [16] & Helper & 日本东北大学 & 2006 & 双臂 14 自由度, 末端配置六维力/力矩传感器, 万向轮式移动, 装载视觉、超声传感器 \\
\hline & [17] & Justin & 德宇航 & 2009 & 全身 51 自由度，两臂采用 DLR 轻量化手臂，关节装载力矩传感器，头部装有视觉传感器 \\
\hline & [18] & Cody & $\begin{array}{c}\text { 乔治亚洲科技 } \\
\text { 学院 }\end{array}$ & 2010 & 双臂 14 自由度, 面向医疗服务, 全方位移动, 装载视觉、位置测量传感器 \\
\hline & [19] & PR2 & Willow Garage & 2015 & 双臂 14 自由度, 4 轮移动平台, 内部装有激光测距仪、触觉传感器等 \\
\hline & [20] & Momaro & 波恩大学 & 2016 & 双臂 14 自由度, 4 条轮式腿, 全方位移动, 内部装载激光扫描仪、广角摄像机等传感器 \\
\hline \multirow{4}{*}{$\begin{array}{l}\text { 足 } \\
\text { 式 }\end{array}$} & [21] & Asimo & 日本本田 & 2003 & 双臂 14 自由度, 重 $54 \mathrm{~kg}$, 电动机驱动, 腕部配置六维力传感器 \\
\hline & [22] & Altas & $\begin{array}{c}\text { 美国波斯顿动 } \\
\text { 力学 }\end{array}$ & 2014 & 双臂 14 自由度, 重 $175 \mathrm{~kg}$, 液压驱动, 内部配置激光测距仪和双闭环视觉系统 \\
\hline & [23] & Chimpw & $\begin{array}{l}\text { 卡内基梅隆大 } \\
\text { 学 }\end{array}$ & 2015 & 双臂 14 自由度, 重 $201 \mathrm{~kg}$, 电动机驱动, 足部装有履带爬行机构, 腕部配备六维力传感器 \\
\hline & [24] & Hubo-II & $\begin{array}{c}\text { 韩国科技先进 } \\
\text { 研究院 }\end{array}$ & 2015 & 双臂 14 自由度, 重 $45 \mathrm{~kg}, 2015$ 年 DARPA 机器人挑战赛冠军, 配置视觉、力传感器等 \\
\hline
\end{tabular}

相关标准化机构结合众学者研究成果制定安全 准则, 其中最具影响力的当属国际标准化组织 (International Organization for Standardization, ISO) 和美国国家标准协会(American National Standards Institute, ANSI)。针对交互安全问题, ISO 最初发布 文件 ISO $10218^{[35-36]}$, 该文件由 “机器人” 和 “机 器人系统与集成” 两部分组成, 分别从机器人结构 设计、控制、应用、维护等多方面制定安全行为准 则及技术要求, 并简要概述了安全协作操作的潜在 方法, 例如对运行速度和距离监测、运行功率和作 用力等因素采取限制措施。为完善机器人发展所面 临的新型安全问题, ISO 又于 2014 年颁布 ISO 13482-2014, 该标准主要对新型个人护理机器人安 全规范进行诠释。2016 年, ISO 针对 ISO 10218 技 术行为规范进行补充, 随附技术规范 ISO/TS $15066^{[38]}$ 。该技术规范主要针对新型协作机器人, 提供了 ISO 10218 所要求的附加信息和细节, 其中 包括生物力学定量限制, 如身体各部位允许的峰值
力或压力; 以及速度和距离监测方程等, 用以表征 安全与生产力之间的关系。该细则规范得到了欧盟 多国的使用。

其中, 速度和距离监测主要由最小安全距离决 定，该计算由 ISO13855 至 ISO/TS15066 逐渐得到 完善, 如下所示

$$
\begin{gathered}
S=v T+C \\
S=v_{H} T_{R}+v_{H} T_{s}+v_{R} T_{R}+B+C+Z_{R}+Z_{S} \\
S\left(t_{0}\right) \geqslant\left(\int_{\tau=t_{0}}^{\tau=t_{0}+T_{R}+T_{S}} v_{H}(\tau) \mathrm{d} \tau\right)+\left(\int_{\tau=t_{0}}^{\tau=t_{0}+T_{R}} v_{R}(\tau) \mathrm{d} \tau\right) \\
+\left(\int_{\tau=t_{0}+t_{R}}^{\tau=t_{0}+T_{R}+T_{S}} v_{S}(\tau) \mathrm{d} \tau\right)+\left(C+Z_{S}+Z_{R}\right)
\end{gathered}
$$

式中, $C$ 表征最小安全保护距离, $v$ 表示人体移动 速度, $T$ 为机器人系统停止时间, $Z_{S}$ 和 $Z_{R}$ 分别表示 传感器感知人员与机器人的位置误差。

此外, 美国标准手册 ANSI R15.06-1999 ${ }^{[39]}$ 于 1999 年公开, 于 2009 年得到重申, 后又于 2012 年 进行修订形成 ANSI R15.06-2012 ${ }^{[40]}$ 。该标准于 2013 
年 3 月 28 日获得批准, 着重对于工业机器人和机器 人系统的制造与集成提供指导方针, 强调安全性、 风险评估的重要性和建立人员安全。该标准在美国 和世界其他国家被广泛使用。

对于上述安全标准的制定, 机器人产业日益普及, 其功能行为愈来愈趋于人类, 为此 VILLARONGA ${ }^{[41]}$ 基于 ISO 13482-2014 规范开始呼吁人类建立关于机 器人分类明确地法律法规。通过结合学术、工业研 究组织和标准化组织的努力, 进而开发更多合适的 相关标准和指标, 完善 ISO 标准的修订和健全相关 法律法规。

\section{3 人机安全交互技术与方法}

新一代机器人与人共享相同空间, 物理接触不 可避免, 基于无意识的意外碰撞与有意图的安全交 互应运而生。根据 pHRI 行为发生流程, HADDADIN 将其分为 7 个步骤: 预碰撞、碰撞检测、分离、识 别、分类、响应以及碰撞后处理, 如图 2 所示 ${ }^{[42]}$ 。 为提高有意交互性能, 降低意外碰撞伤害, 本节围 绕人机交互功能特性, 结合 pHRI 和 sHRI 实现方法, 从接触前预警、接触后感知和行为决策等方面加以 补充和阐述。

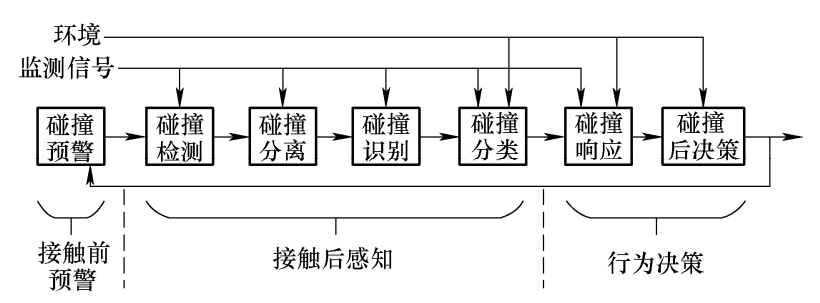

图 $2 \mathrm{pHRI}$ 行为发生流程与响应概述图

\section{1 接触前预警}

接触前预警旨在完成非结构环境下的人机共存 问题, 避免不必要碰撞发生。与传统静态、结构化 环境不同, 多模态非结构环境要求机器人具有对环 境实时感知能力, 并及时规划作业轨迹, 避免碰撞。 传统碰撞避免方法多基于 $\mathrm{C}$ 空间方法与人工势场 法。C 空间法以机器人关节空间建立 $\mathrm{C}$ 空间, 将静 态障碍物映射到该空间下组成障碍空间, 通过在障 碍空间外的自由空间进行路径规划, 寻找最优避碰 轨迹。现有研究将 $C$ 空间方法分为几何法 ${ }^{[43-44]}$ 和拓 扑法 ${ }^{[45]}$ 等; 人工势场法则通过定义引导机器人运动 的排斥矢量场, 根据动态变化的环境因素修改其轨 迹, 从而允许更复杂的安全行为。

相比 $\mathrm{C}$ 空间方法, 人工势场法具有动态避障特 性, 并在相继研究中得到了进一步改进。在近期研 究中, YANG 等 ${ }^{[46]}$ 提出一种改进策略进行优化吸引
和排斥势场，克服了传统人工势场规划易陷入局部 最小值缺陷; LUCA 等 ${ }^{[47]}$ 分别针对机器人末端执行 机构和机器人其他部位建立两种控制架构, 并将监 测距离和速度等因素所生成的人工势场危险指数嵌 入安全控制系统; ZHANG 等 ${ }^{[48]}$ 采用新型势能函数 代替传统人工势场来指导路径规划避免碰撞, 该方 法规避了规划过程中繁琐的运动学计算, 降低了算 法运算复杂度; POLVERINI 等 ${ }^{\left[{ }^{49]}\right.}$ 于 2014 年开发了 基于动静态安全场、运动学层面的安全评估, 其主 要特点在于解释了危险源与计算场间的相对运动; LASOTA 等 ${ }^{[50]}$ 通过分析危险源形状或大小对于安 全场影响因素, 并将其引入 YuMi 实时控制器加以 验证, 表明该势场可用于防止双臂自碰撞和机器人 与人碰撞。

随着机器人逐渐向着开放性空间发展, 越来越 多的机器人采用视觉信息处理及技术。突破传统静 态、固定安全区域限制, RYBSKI 等 ${ }^{[51]}$ 结合多传感 器信息融合技术, 基于立体摄像机采集工作单元数 据, 系统检测环境中的人员与机器人, 并根据本体 位置和轨迹生成动态危险区域; FLACCO 等 ${ }^{[52]}$ 基于 深度传感器建立深度空间方法, 通过估计机器人与 静态和移动障碍物间的距离, 进而将这种实时距离 测量及障碍物速度估计信息与基于排斥力矢量的控 制器一起用作防撞技术; AZORÍN-LÓPEZ 等 ${ }^{[53]}$ 基 于行为预测和图像数据信息, 通过 RGB 输入的数据 和标准化活动描述矢量(Activity description vector, ADV)来消除对定时或时间序列信息的需要, 进而预 测人体活动行为, 避免碰撞; 针对视觉采集过程中 可能存在的物体遮掩问题, AVANZINI 等 ${ }^{[54]}$ 基于机 载传感方法, 开发了一种分布式距离传感器, 并优 化了机器人本体传感器最佳装配位置, 一定程度上 降低了非结构环境下碰撞事件的发生。

除此之外, 部分学者采用速度和位置监测方法, 适时放缓运行速度或者停止工作, 实现碰撞避免或 伤害最小化。如 VOGEL 等 ${ }^{[5]}$ 基于投影仪映射机器 人关节位置和速度动态变化域, 检测调控进入该虚 拟安全区域的合适时间; LASOTA 等 ${ }^{[56]}$ 开发一种安 全系统, 通过快速感知并计算人体与机器人间距离, 调控相关函数参数以降低机器人运行速度。

上述碰撞预警方法着重强调基于潜在场及安全 区域与人员距离逐渐减慢机器人运动, 这极大程度 限制了机器人运动灵活性。此外视觉预警多受监控 盲区、环境复杂考量、处理算法效率等限制。对此, 在动态、非结构环境下的机器人工作效率与安全保 证的权衡性策略研究, 以及基于多传感器信息融合 的实时碰撞避免方法依然是热点开放性课题。 


\section{2 接触后感知}

接触后感知旨在完成不确定因素下的人机交互 信息感知, 实现意外碰撞伤害最小化和自主交互效 率最大化。根据安全因素, 包括机器人类型、传感 系统和任务分配等。严格的避碰并不总是可行, 诸 如精密装配、打磨等特殊任务必然需要一定程度的 物理接触, 并且传统的位置控制模式已不足以满足 当前特定任务需要。尤其在人机协作、康复护理、 医疗手术等机器人中, 要求机器人引入力反馈控制 模式，具备力感知能力和柔顺特性。

\subsection{1 力感知能力}

力感知能力包括碰撞检测, 接触力位置、大小 和方向矢量的识别, 接触意图感知等。碰撞检测目 的在于快速、准确感知机器人是否发生碰撞, 其关 键在于检测阈值的选取; 接触力的信息识别目的在 于让机器人理解环境约束力信息, 从而决断更好交 互策略; 接触意图的感知旨在理解人类接触意图, 类似状态转换机, 实现安全交互模式转换及行为决 策。HADDADIN ${ }^{[42]}$ 通过对 DLR 以往研究成果总结 和扩展, 着重对于碰撞检测、分离和识别做出阐述, 并指出基于动量观测器的信号监测方法是当前机器 人最好的交互力感知技术。该方法不仅可以避免加 速度以及惯性逆矩阵计算所引入的噪声问题, 且具 有解耦特性, 对于碰撞检测、分离和识别统一适用。 此外, 对于传统的基于电流、力矩传感器、逆动力 学、能量观测器等监测方法做出了详细比较, 关于 该方面本文不再进行赘述。

机器人系统是一个高度非线性强耦合系统, 其 关节自身存在摩擦、间隙误差以及由柔性所产生振 动等非线性因素, 严重影响机器人重复定位精度及 关节力矩感知信息的准确性(图 3), 其作为机器人研 究的焦点研究问题经久不衰。为提高机器人准确力 感知能力及 $\mathrm{pHRI}$ 安全性, 大量文献针对机器人系 统非线性不确定因素进行研究, 大致分为基于模型 和无模型方法。基于模型的方法常局限于系统模型 的建立和关键项参数的辨识等问题。关于柔性关节 机器人(Flexible joint robots, FJR)建模与控制问题可 以参考 SPONG 等 ${ }^{[57-58}$ 的贡献。此外, $\mathrm{KHAN}^{[59]}$ 着 重对摩擦力因素进行了详细的回顾与分析。

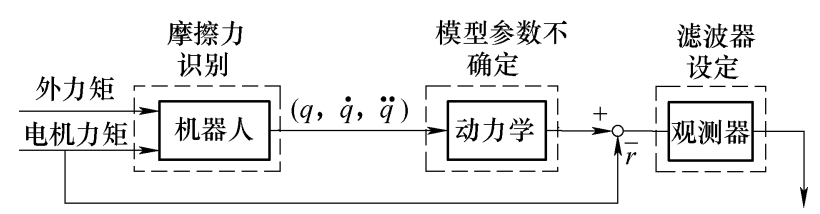

图 3 基于模型的力感知原理图

针对模型参数辨识问题, 多围绕系统函数方程 作为先验模型, 结合最小二乘法、自适应算法或卡
尔曼相关算法等进行参数辨识, 以提高模型准确 性, 增强基于模型控制的安全交互能力。例如 $\mathrm{FLACCO}^{[60-61]}$ 通过设计系统残差估计辨识方法, 间 接估计观测关节扭矩, 避免了附加传感器的使用以 及高阶微分噪声; 随后 MÉNARD ${ }^{[63-63]}$ 提出基于调 制函数和操作算子的参数辨识方法, 基于动力学模 型中各位置多阶积分项间的转换, 获取低阶微分项 可靠辨识; JIA 等 ${ }^{[64]}$ 基于统计学框架通过改进激励 轨迹优化算法, 规避优化过程易陷入局部最优问题, 基于加权最小二乘提高了系统辨识鲁棒性且提高了 辨识精度; JIN 等 ${ }^{[65]}$ 结合自适应控制算法提出一种 动态参数辨识方法, 有效解决了动态变负载等不确 定任务下的参数变化问题; LICHIARDOPOL ${ }^{[66]}$ 基于 机器人动力学知识, 针对未知负载以及外力扰动, 提出鲁棒非线性扰动估计辨识方法, 使得在准静态 时变和不确定载荷扰动下, 可以对低频外力进行鲁 棒估计; WAHRBURG ${ }^{[67-69]}$ 仅通过采集电动机电流、 位置和速度信号，基于自适应和卡尔曼滤波方法对 于模型进行参数辨识, 避免了计算惯性逆和加速度 噪声问题; 并且作者针对 7 自由度冗余解进行优化 以获取最优轨迹, 进一步提高笛卡儿力与力矩估计 准确性, 增强力感知能力; $\mathrm{HU}^{[70]}$ 针对模型参数不确 定问题提出一种半参数模型方法, 基于刚体动力学 理论对机器人动力学模型进行识别, 其次采用了多 层感知训练器对刚体动力学模型进行非参数补偿, 进而采用扰动卡尔曼滤波对于系统动量残差值进行 观测与估计, 提高力感知能力。

对于无模型力感知, 多采用智能算法与高斯噪 声、灰盒等估算方法。如 MAKAROV 等 ${ }^{[71-72]}$ 基于 灰盒模型, 将残差矢量分解为关于外力矩、模型未 知量以及摩擦力等因素项, 通过自适应算法分离各 不确定项对于碰撞感知的影响，进而设定动态阈值 用以匹配碰撞检测最佳灵敏性。然而该方法仅适用 末端接触, 并未解决机器人任意位置力感知问题; SHARKAWY ${ }^{[73]}$ 基于 $\mathrm{LM}$ 算法构建多层神经网络以提 高人机碰撞检测能力。该方法无需动力学模型先验知 识, 也无需额外传感器。相比其他训练方法, 其用更 少的迭代次数和更短的时间来完成更大数值的设置 与收玫, 算法稳定且迅速; INDRI ${ }^{[74-75]}$ 基于电流监测 方法, 通过有限状态机(Finite state machine, FSM)建立 轻型和重型机械臂统一适用的虚拟碰撞传感器。该方 法主要采用刚性模型, 且采用本体感知避免额外传感 器所引入噪声等问题, 但未考虑关节柔性因素等。

此外, 由系统非线性因素所导致的碰撞检测漏 报与误报情形(原理如图 4 所示)得到诸多学者关注。 SOTOUDEHNEJAD ${ }^{[76-77]}$ 基于机器人模型详细的函 
数表达, 提出机械手关节空间中观测残差的时变阈 值, 以提供比恒定阈值更精确的碰撞检测能力; JUNG 等 ${ }^{[78]}$ 设计一种带通滤波器方法, 过滤噪声影 响; 随后又基于频域范围, 采用傅里叶级数变换思 想, 将机器人动力学模型简化为常数项与三角函数 项, 进一步分析设计频率带宽上界, 将频率边界在 频域中的位置趋势应用于变频窗观测, 进而减小模 型误差所导致的错误检测 ${ }^{[79]}$; $\mathrm{HO}$ 等 ${ }^{[80]}$ 提出了一种 基于带通滤波的碰撞检测算法。利用巴特沃斯二阶 带通滤波器(Butterworth 2nd-order BPF) 有效地去除 机器人自身运动产生的转矩, 保留碰撞产生的转矩 用于碰撞检测。这提高了算法对模型不确定性的鲁 棒性, 同时该算法避免使用加速度信息所产生二阶 微分噪声等, 然而滤波器的使用容易引入延时项。

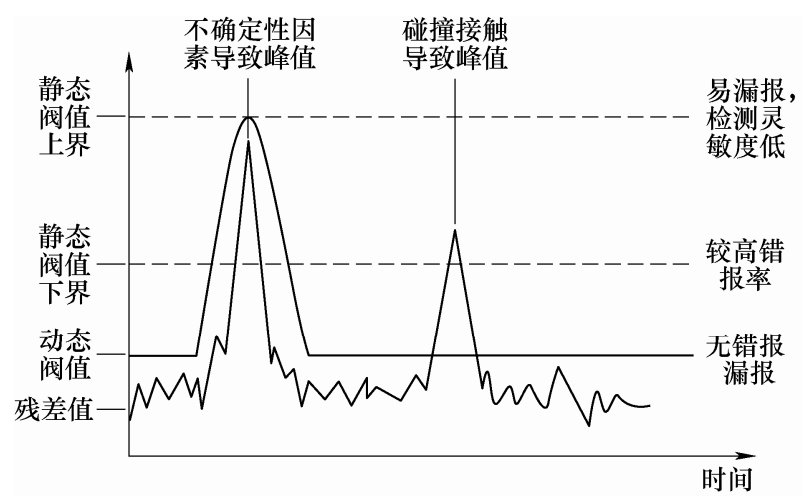

图 4 动、静态阈值与碰撞检测的影响

针对碰撞意图感知, 诸多学者采用机器人本体 信息感知人类与机器人接触意图。ERDEN 等 ${ }^{[81}$ 基 于位置控制系统的零动量守恒原理, 提出了一种基 于固定位置参考的纯阻抗控制模式和基于人工意图 的交互控制模式之间的切换方案, 机器人通过观察 控制力的变化来判断人类接触意图; $\mathrm{CHO}$ 等 ${ }^{[82]}$ 基于 碰撞过程中外力的变化速度与预期接触时的变化速 度差异, 进而观测区分意外碰撞和意图交互; GERAVAND $^{[83]}$ 假设意外碰撞产生高频信号且有意 接触产生低频信号, 通过在电动机电流上同时并联 高通和低通滤波器来执行转换开关, 通过比较滤波 后的信号与时变阈值, 进而区分有意与无意接触; LI 等 ${ }^{[84]}$ 提出一种自适应阻抗控制方法, 并基于神经 网络构建在线估计方法, 用以解决人体肢体模型估 计的非线性和时变特性, 进而定义人体肢体模型中 所期望轨迹为人体运动意向, 并集成到控制系统中; GOLZ 等 ${ }^{[85}$ 利用机器学习结合物理接触模型, 进而 通过观察实际接触数据推导出用于非线性支持向量 机(Support vector machine, SVM)的分类特征, 通过 模拟和物理试验结果, 分类器能够在线准确地区分 有意和无意碰撞等。
尽管针对机器人不确定性因素下的力感知问题 已得到有效解决, 然而诸如控制延时、模型参数精 准辨识、谐波反向传递电流信息感知、力传感器成 本等问题依然掣肘机器人感知性能发展; 并且当前 研究多集中于单点接触, 面对多点碰撞分离与识别 问题有待于进一步加强研究。随着现代数学日益发 展, 微分流形、旋量理论在机器人学应用愈来愈普 遍, 结合状态观测及预测控制等方法, 面对人机交 互中的环境结束问题, 有望基于空间矢量建模方法 得到进一步解决与优化。

\section{2 .2 柔顺特性}

柔顺特性多集中于缓解冲力、释放撞击能量、 减小碰撞伤害以提高安全性能, 其主要表征为被动 柔顺和主动柔顺两个方面。被动柔顺基于机器人结 构设计, 通过降低机器人有效惯性质量、减小接触 刚度等方法实现柔顺行为。早期被动柔顺多采用末 端柔顺装置, 如 RCC 装置, 现常用于装配任务 ${ }^{[86]}$ 。 在近期的研究中, 被动柔顺多集中于基于耦合弹性 驱动(Coupled elastic actuation, CEA)、串联离合驱动 (Series clutch actuator, SCA)、变阻抗驱动(Variable impedance actuation, VIA)、串联弹性驱动 (Series elastic actuation, SEA)、变刚度驱动(Variable stiffness actuation, VSA)等研究, 并围绕相关驱动进行不同控 制算法的性能验证。其基本特性如表 2 所示。此外, GREBENSTEIN 针对 DLR 系列机器人研发一种对 抗式张紧驱动手爪 ${ }^{[93]}$, 该手爪的尺寸大小和重量接 近人类原型, 尤其在抓取性能、鲁棒性和动力学上 更加符合实际人手，进一步提高了人机交互灵活性 与安全性能。

表 2 典型被动柔顺关节概述

\begin{tabular}{|c|c|c|}
\hline $\begin{array}{l}\text { 柔顺关 } \\
\text { 节类型 }\end{array}$ & 文献 & 原理与特性 \\
\hline CEA & {$[87]$} & $\begin{array}{l}\text { 在电动机与连杆之间引入 } 2 \text { 个并列弹性组件, 分 } \\
\text { 别具有低、高刚度特性, 通过切换高低速运行状 } \\
\text { 态下两个刚度组件的配合作用, 实现人机交互伤 } \\
\text { 害始终保持在较小程度。 }\end{array}$ \\
\hline SCA & {$[88]$} & $\begin{array}{l}\text { 基于笛卡儿空间力约束, 在关节建立可调力矩限 } \\
\text { 制器, 并通过设置机器人工具中心点安全静力矩 } \\
\text { 阈值, 增强人机交互安全性, 但是对于安全阈值 } \\
\text { 的选取要求较高。 }\end{array}$ \\
\hline VIA & [89] & $\begin{array}{l}\text { 在关节中引入模块化弹簧-阻尼单元, 通过改变单 } \\
\text { 元中的弹簧的形变量, 进而将力控制转化为基于 } \\
\text { 弹簧形变的位置控制, 实现变阻抗驱动。 }\end{array}$ \\
\hline SEA & {$[90]$} & $\begin{array}{l}\text { 在关节中串联齿轮与机械弹簧(包括扭簧、压簧 } \\
\text { 等), 实现冲击载荷低通滤波功能, 降低碰撞冲击 } \\
\text { 力, 该结构增加了系统控制难度。 }\end{array}$ \\
\hline VSA & $\begin{array}{l}{[91]} \\
{[92]}\end{array}$ & $\begin{array}{l}\text { 关节采用大小双电动机力矩输出模式, 大电动机 } \\
\text { 低速大扭矩控制连杆转动, 小电动机高速小扭矩 } \\
\text { 调节杆件刚度变化。结构较复杂, 成本较高, 且 } \\
\text { 导致控制结构比较复杂。 }\end{array}$ \\
\hline
\end{tabular}


主动柔顺控制主要解决机器人力与位置间的权 衡问题。机器人操作过程可大致分为无约束空间下 的自由运动、环境约束下的运动以及接触过程中两 种运动状态间的转换。从机器人运动控制特点来看, 可归结为以下几类: 力/位置混合控制 ${ }^{[94]}$ 、基于力反 馈的阻抗控制、基于位置反馈的导纳控制 ${ }^{[95]}$ 等。此 外结合智能控制算法, 如自适应控制、鲁棒控制、 模糊控制、神经网络控制等, 进一步加强机器人系 统对于系统非线性扰动鲁棒特性, 提高了运动控制 性能与交互安全性。

由于力/位置混合控制在自由空间与约束空间 下模式转化呈现非连续性, 必须根据精确的环境约 束方程来反映任务要求, 进而确定精准雅克比矩阵 来确定力和位置控制方向, 这将很大程度增加非结 构环境建模成本。之前的研究表明 ${ }^{[96-98]}$, 阻抗控制 可以统一自由空间和约束空间的控制, 并且对外界 扰动和不确定性具有良好的鲁棒性, 更加适用于 交互任务。但是上述研究主要解决笛卡儿空间内 的交互问题, 针对零空间下的圥余协作机器人交 互问题仍然面临挑战(图 5)。文献[99]提出一种局 部任务放松策略, 利用机器人几余度将反应力矩 投射到动态任务矩阵的零空间, 从而不影响初始 末端执行机构的运动, 形成动态一致的几余解决 方法; SADEGHIAN 等 ${ }^{[100]}$ 关注了零空间的阻抗行 为, 但是设计的非线性控制器忽略了关节在笛卡儿 空间的位置误差; 文献[101]基于机器人任务空间误 差和广义动量设计了两种不同控制观测器算法, 估 计和补偿作用于任务变量的广义力, 避免使用关节 力矩测量, 从而保证任务空间误差的渐近稳定性, 提高了零空间阻抗控制效果。

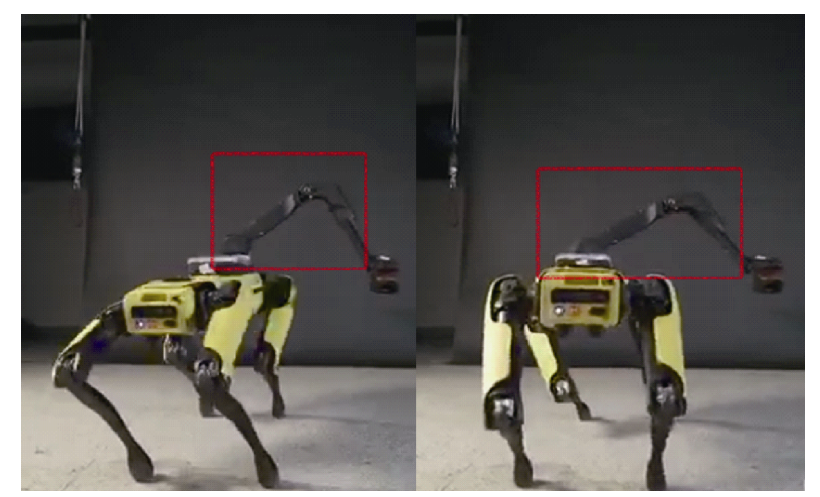

图 5 SpotMini 零空间状态下舞姿

\section{3 行为决策}

区别于人机共存下的单向交互行为, 行为决策 旨在完成人机自主交互、双向协同问题, 实现拟人 脑决断决策行为, 以保障人机协作任务同时兼具机 器人理解并跟随复现人体活动行为特征。区别于传
统示教方法, 行为决策贯穿交互始终, 强调机器人 主动认知与交互学习, 通过人员行为预测和运动意 图感知来实时生成路径以增强双向安全交互响应。 通常简单 HRI 环境可视为静态或准静态, 通过组合 任务和运动规划来实现有效遍历复杂的搜索空间, 以及利用约束的分层编码来确保 HRI 安全性。与传 统基于规划成本函数考量规划方法不同，未来 HRI 面向复杂动态环境, 基于快速变化当前状态配置以 实现连续重新规划, 其过程包括当前状态推理, 以 及未来任务和动作预测等, 多依赖于以手势、表情、 语音等视觉、听觉信息为底层传感器输入的 sHRI, 融合机器学习等人工智能技术, 实现人机自主安全 交互。

\subsection{1 人体行为感知}

实现动态环境下的人机安全交互响应，依赖于 交互成员间良好认知, 适时、实时产生交互行为(诸 如交互切入时刻、交互行为响应等), 其实现方法可 归纳为行为预测以及行为学习。根据多模态环境下 的人类活动行为, $\mathrm{RYOO}^{[102]}$ 于 2011 年提出一种预 测方法, 该方法基于 RGB 视频连续输入帧中的视觉 词导出关于人类行为的时空特征积分直方图, 通过 分析帧中发生显著运动的点以及总结帧中相应部分 的梯度来计算这些点的描述, 进而确定这些视觉信 息。随后 RYOO 采用聚类算法对该方法所生成相似 特征组与人类预测行为进行了检验匹配, 进而确定 安全行为, 并于 2015 年做了进一步改进 ${ }^{[103]}$; KOPPULA 等结合 RGB-D 输入和对象可供性来感 知并预测未来的人类行为 ${ }^{[104]}$ 。其主要通过预期的时 间片段扩充条件随机场(Conditional random fields, CRF), 形成预期的时间条件随机场, 进而计算该场 分布来进行预测; MAINPRICE 等利用到达标记点 的动作演示来生成预测工作空间占用模型 ${ }^{[105]}$ 。其针 对特定任务中的目标位置训练单独高斯混运动混合 模型(Gaussian mixture models, GMMs), 并使用高斯 混合回归(Gaussian mixture regressions, GMRs)生成 抵达标记位置运动轨迹。后基于对新到达运动的初 始片段和计算的 GMMs 和 GMRs 的观察, 进而计算 个体在模拟共享工作区中的占用可能性, 随即选择 动作和路径, 尽量减少预期由人类占据的工作空间 区域的不合理侵入; PÉREZ-D'ARPINO 等的工作 ${ }^{[106]}$ 类似于文献[105], 但他们采用时间序列分析, 系统 使用学习模型在运动初始阶段执行贝叶斯分类, 以 便预测人将到达位置并选择干扰最小的机器人动 作。该模型考虑了沿运动轨迹的点序列, 在人类运 动过程的早期具有更好的可辨性和更高的分类置信 度等。 
针对交互系统中的人体行为感知, 上述方法多 基于传感器数据预测推理, 而拟人化决策常表征为 记忆认知与行为再现。对此, DOMINEY ${ }^{[107]}$ 提出了 一种直接的任务模型推理方法, 该方法引入了交互 历史来促进预期的机器人行为, 系统针对协同组装 任务, 将当前操作序列与先前观察到的序列进行比 较, 以确定当前交互是否为先前观察到的任务序列 的实例; NIKOLAIDIS 等 ${ }^{[108]}$ 探索了另一种利用概率 框架编码人机协作任务并利用结果预测人类行为的 方法。该方法中, 协作任务被编码为一个马尔可夫 决策过程(Markov decision process, MDP), 试验结果 表明马尔可夫链的熵率变化可被用于确定协作过程 中的人类成员下一步将机器人执行何种操作; 随后 NIKOLAIDIS 等又将 MDP 扩展为混合可观测马尔 可夫决策过程(Mixed observability Markov decision process, MOMDP $)^{[109]}$ 。其通过联合行动演示自动学 习用户模型, 基于反向强化学习对每个群集的动作 序列和学习奖励函数进行聚类。然后机器人使用该 模型来预测用户类型, 从而预测用户的行为并执行 适当的预期行为。结果表明, 与语音指令、手动控 制机器人相比, 在任务执行时间和人类空闲时间等 量化指标上, 该模型都展现出较好性能。

此外, LI 等基于概率后缀树制定人体动作间的 变量阶马尔可夫模型 ${ }^{[110]}$, 用于预测具有较长连续时 间特性组成的复杂连续行为, 进而解决动作因果关 系。其提出的框架还基于预测累积函数(Predictive accumulative function, PAF), 通过数据强化学习来进 一步确定行为的可预测性, 实现顺序时间序列下的 动作共现。

\subsection{2 运动意图识别及环境感知}

针对具体任务下人体动作意图及动作特征则主 要依赖于人体适当运动模型, 利用诸如生物力学 预测分析或自然运动特征推理等技术。在缺乏明 确目标位置, 或者对于其动作预测难以实现的应 用, 常基于运动特性的预测, 结合各种运动模型 利用可能目标的预测来评判人体朝向目标运动所 采取的路径。

当基于目标意图预测人体运动时, 系统推断人 类的目标并预测其到达该目标可能采取的路径或轨 迹。如文献[104]的工作, 系统预测到人体动作发生, 随即采用 Bézier 曲线来定义人手在执行动作时的潜 在轨迹; JIANG 等扩展了该项研究 ${ }^{[111]}$ 。其通过高斯 过程计算人体运动高维模型的低维表示, 形成高斯 过程潜在条件随机场模型。通过学习高维和低维表 示间的相互映射, 实现运动的感知预测及推理学习; MAINPRICE 等 ${ }^{[112]}$ 基于动作捕捉系统收集执行共同
操作任务的两位工作者的示例数据, 进而将收集的轨 迹以及编码平滑度和距离关系的特征函数用作路径 积分逆强化学习(Path integral inverse reinforcement learning, PIIRL)算法输入, 以便产生到达运动成本 函数。该方法基于成本函数和具有 23 自由度的人体 运动模型, 通过运动规划 (Stochastic trajectory optimization for motion planning, STOMP)算法的随 机轨迹迭代重新计算运动, 进而识别人体运动。

针对人体动作特征的识别与学习, TAKANO 等 ${ }^{[113]}$ 基于骨骼运动数据编码(Hidden Markov model, HMM), 通过 Ward 聚类形成 “运动符号树” 分层 结构。结合 Ngrams 整合运动符号序列, 以形成有 向 “运动符号图”, 表示人类行为间的因果关系。进 而将运动符号图与当前运动观察结合使用以预测将 来运动模式; XIAO 等 ${ }^{[114]}$ 基于人类轨迹先验信息来 训练 SVM 分类器, 结合 PAM 算法将分类聚类, 并 依赖可修改性距离函数更好地聚类非重叠运动轨 迹, 进而匹配观察轨迹实现运动感知。该方法优势 在于基于无监督学习方式, 所生成的新轨迹可进一 步用来优化预测和调整运动; KUDERER 等 ${ }^{[115]}$ 基于 人体运动演示来开发一种学习关节轨迹的运动预测 方法。研究人员基于最大熵原理, 考虑到达目标所 需时间量、加速度曲线、动作速度等特征, 分析并 学习人与人之间相互作用轨迹及动作特征。该方法 在模拟和物理环境中得到验证, 表明基于关节轨迹 的人类动作识别方法可以自然且兼容的方式成功地 引导机器人。此外, WALKER 等 ${ }^{[116]}$ 基于无监督的 数据驱动方法, 实现对静态图像的视觉感知。该方 法不需要关于活动的先验假设, 通过投影场景中子 部分的位置和外观, 提供对于环境的视觉预测。虽 然该方法不是专门为人体运动感知而设计, 基于环 境感知方法可以很容易地应用于该领域。

尽管上述方法对于预测、学习和再现等行为决 策呈现良好效果, 然而连续规划多步决策受搜索空 间大和参数策略潜在影响, 收玫效率低; 关于逆强 化学习所引入奖励函数, 常附加额外参数, 无疑增 加计算量; 而且对于连续输入空间问题, 现有方法 集中于离散的有限集合, 因此在学习过程中构造学 习系统内部状态和动作空间依然是热点研究问题。 伴随生成式对抗网络(Generative adversarial nets,

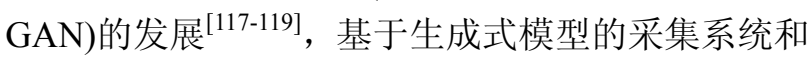
基于判别式模型的决策系统, 可以快速权衡环境中 已知和未知的动态平衡, 加速机器人对于环境信息 的感知与学习。然而其现在主要应用于静态图像的 识别与处理。在机器人动态环境变化中, 结合视觉 处理方法与智能控制, 构建时间序列的 GAN 环境 
感知与生成系统, 实现拟人脑实时决断决策机制面 临挑战。

\section{4 人机安全交互发展趋势}

根据物理人机交互功能特性, 从交互接触前预 警、接触后感知和行为决策等方面, 将非结构化环 境中的人机交互问题分为人机共存、人机单向交互、 人机自主交互与双向协同三个子问题。当前已初步 实现了人机共存与单向交互问题, 然而涉及安全性 的大部分工作集中在碰撞避免和限制系统内存储的 速度或能量, 这在很大程度上牺牲了作业效率; 并 且由于外置传感器可靠性、实时性和噪声等特性因 素, 严格意义上的碰撞避免难以实现; 此外, 机器 人环境感知与控制性能受系统非线性不确定因素影 响严重。因此, 通过机器人本体结构设计、运动控 制与规划以及类人脑预测学习等方法来实现机器人 本质安全最大化, 完成人机自主交互与双向协同依 然是未来热点研究方向。其科学问题可归纳为: 一、 面向非结构动态环境和不确定作业任务下的机器人 适应性规律研究; 二、机器人环境感知与行为理解 机理; 三、人-机-环境多元交互与协同控制机理。 具体涉及以下几点。

（1）结构层面: 设计拟人化灵巧柔顺机械臂; 开展适用于机器人的高能量密度直驱驱动原理研究 与设计; 结合机构学与机器人学发展 ${ }^{[120]}$, 拓展现代 机构设计新理论、新方法; 考量极端环境下机器人 的稳定性与可靠性分析; 针对机器人弹性包裹材料 或电子皮肤 ${ }^{[121]}$ 等, 研究低成本高性能装备材料, 降 低成本同时完善机器人触觉的意外碰撞感知能力。

(2) 柔顺控制层面: 开发稳定、实时控制器; 开展力觉/触觉/滑觉等多位多元信息感知原理研究, 完善基于非线性因素的预测控制与补偿; 针对模型 控制方法, 强化多输入多输出人机交互机理研究, 构建人-机器人-环境多元交互动力学模型, 完善非 结构化环境与不确定性作业任务等复杂系统在线辨 识; 整合变刚度、变阻抗控制思想, 结合人体运动 机理, 基于脑电、肌电等生物信号, 开展拟人化新 型刚体运动力控方法研究。

(3) 规避外置传感器所附加空间占用、成本消 耗及噪声问题, 综合基于模型与无模型控制方法, 基于机器人本体信息开展 $\mathrm{pHRI}$ 分解研究, 实现人 机交互高效碰撞检测、精准碰撞分离、准确碰撞识 别和快速碰撞响应。

(4) 运动规划层面: 规划任务执行时间和并发 运动量, 权衡安全交互行为与工作效率矛盾问题;
构建机器人拓扑运动构态实时规划; 考虑规划遍历 搜索收玫性，优化成本函数与参数潜在影响; 结合 人类运动意图预测，快速实现笛卡尔空间内几何推 理和任务约束计算, 以最大作业效率实现零空间下 人机物安全交互。

(5) 行为技能学习与决策层面: 结合 SHRI 认知 推理规则, 识别时间序列状态下的非结构环境变化 问题, 研究人员行为意图与情绪辨识, 增强对语音/ 手势/情绪等多元信息复合与理解; 突破学习系统在 连续学习过程中内部状态和动作空间构造方法, 加 深机器人功能失效判别与容错规划。

(6) 摒弃传统手动示教学习弊端, 基于人工智 能方法, 融合 sHRI 认知学习, 建立多模态自然交 互技能学习映射, 实时构建人机双向协同行为拓扑 关系; 结合云计算和大数据发展, 逐渐兼具人类运 动行为和类人脑网络决断机制, 实现自主交互、与 人双向协同和协调互补, 提高机器人对广泛任务规 划的协调性及对环境适应性。

（7）社会层面: 完善人体伤害评价标准, 评估 复杂多层安全系统有效性, 包含各种场景和测试用 例, 衡量相关有效性指标, 构建统一人机交互安全 评价准则; 考虑人性、伦理等概念, 规范相关行业 法律法规, 打造未来机器人与大众生活和谐蓝图。

\section{5 结论}

安全交互问题作为机器人融入大众日常生活的 基本要求, 已受到广大学者和企业的青睐。本文以 机器人共性关键技术为基础, 将人机交互行为按时 间序列分解，以安全评价准则为参考标准，从结构 本体、控制、环境感知、运动规划和技能学习等层 面展开阐述，为人机安全交互问题构建了系统的理 论支撑与方法指导；结合当前面临的问题与挑战， 表明人机交互未来发展趋势与内容概述，旨在进一 步完善相关产业技术与学科融合，为 “中国制造 2025 ” 的发展增砖添瓦, 构建新型工业体系和智能 家园蓝图。

\section{参 考 文 献}

[1] SHEIKHOLESLAMI S, MOON A J, CROFT E A. Cooperative gestures for industry: Exploring the efficacy of robot hand configurations in expression of instructional gestures for human-robot interaction[J]. The International Journal of Robotics Research, 2017， 36(5-7): 699-720.

[2] PORTUGAL D, ALVITO P, CHRISTODOULOU E, et al. A study on the deployment of a service robot in an elderly care center[J]. International Journal of Social 
Robotics, 2019, 11(2): 317-341.

[3] DE STEFANO M, MISHRA H, BALACHANDRAN R, et al. Multi-rate tracking control for a space robot on a controlled satellite: A passivity-based strategy[J]. IEEE Robotics and Automation Letters, 2019, 4(2): 1319-1326.

[4] 王田苗, 陶永. 我国工业机器人技术现状与产业化发展 战略 [J]. 机械工程学报, 2014, 50(9): 1-13.

WANG Tianmiao, TAO Yong. Research status and industrialization development strategy of Chinese industrial robot[J]. Journal of Mechanical Engineering, 2014, 50(9): 1-13.

[5] 王国彪, 陈殿生, 陈科位, 等. 仿生机器人研究现状与 发展趋势[J]. 机械工程学报, 2015, 51(13): 27-44.

WANG Guobiao, CHEN Diansheng, CHEN Kewei, et al. The current research status and development strategy on biomimetic robot[J]. Journal of Mechanical Engineering, 2015, 51(13): 27-44.

[6] 高峰, 郭为忠. 中国机器人的发展战略思考 [J]. 机械工 程学报, 2016, 52(7): 1-5.

GAO Feng, GUO Weizhong. Thinking of the development strategy of robots in China[J]. Journal of Mechanical Engineering, 2016, 52(7): 1-5.

[7] KEBRIA P M, AL-WAIS S, ABDI H, et al. Kinematic and dynamic modelling of UR5 manipulator[C]// 2016 IEEE International Conference on Systems, Man, and Cybernetics (SMC), 9-12 Oct. 2016, Budapest, Hungary. IEEE, 2016: 4229-4234.

[8] BAHRIN M A K, OTHMAN M F, AZLI N H N, et al. Industry 4.0: A review on industrial automation and robotic[J]. Jurnal Teknologi, 2016, 78(6-13): 137-143.

[9] Rethink Robotics. Sawyer-redefining robotics and manufacturing — rethink robotics[EB/OL]. USA: Rethink Robotics [2019-02-20]. Available : http : //www.rethinkrobotics.com/sawyer3.

[10] GUIZZO E. Employee of the month. Every month[Top Tech 2017][J]. IEEE Spectrum, 2017， 54(1): 34-35.

[11] IREGUI S, DE SCHUTTER J, AERTBELIËN E. Constraint-based robot control for BMI applications[C]// 38th Benelux Meeting on Systems and Control, 19-21 Mar. 2019, Lommel, Belgium. 2019: 159-159.

[12] SUGIHARA T, NAKAMURA Y. Whole-body cooperative balancing of humanoid robot using COG Jacobian[C]// 2002 IEEE/RSJ International Conference on Intelligent Robots and Systems, 30 Sept.-4 Oct. 2002, Lausanne, Switzerland, Switzerland. IEEE, 2002 : 2575-2580.

[13] SHAURIA R L A, SAIKIB K. Sensor integration and fusion for autonomous screwing task by dual-manipulator hand robot[J]. International Symposium on Robotics and
Intelligent Sensors 2012(IRIS 2012), 41: 1412-1420.

[14] CREMER S, MASTROMORO L, POPA D O. On the performance of the Baxter research robot[C]// 2016 IEEE International Symposium on Assembly and Manufacturing (ISAM), 21-22 Aug. 2016, Fort Worth, TX, USA. IEEE, 2016: 106-111.

[15] KIRSCHNER D, VELIK R, YAHYANEJAD S, et al. YuMi, come and play with Me! A collaborative robot for piecing together a tangram puzzle $[\mathrm{C}] / /$ International Conference on Interactive Collaborative Robotics. Springer, Cham, 2016: 243-251.

[16] HISAO T, HITOSHI N, YOSHINORI $\mathrm{K}$, et al. Recognition of objects indicated by deictic pronouns for helper robots[C]// 2006 SICE-ICASE International Joint Conference, 18-21 Oct. 2006, Busan, South Korea. IEEE, 2006: $1437-1440$.

[17] FUCHS M, BORST C, GIORDANO P R, et al. Rollin'Justin-design considerations and realization of a mobile platform for a humanoid upper body[C]// 2009 IEEE International Conference on Robotics and Automation, 12-17 May 2009, Kobe, Japan. IEEE, 2009: 4131-4137.

[18] JAIN A, KEMP C C. Pulling open doors and drawers: Coordinating an omni-directional base and a compliant arm with equilibrium point control[C]// 2010 IEEE International Conference on Robotics and Automation, 3-7 May 2010, Anchorage, AK, USA. IEEE, 2010: 1807-1814.

[19] NIMA R, MARY-ANNE W. Smooth robot motion with an Optimal Redundancy Resolution for PR2 robot based on an analytic inverse kinematic solution[C]// 2015 IEEE-RAS 15th International Conference on Humanoid Robots(Humanoids), 3-5 Nov. 2015, Seoul, South Korea. IEEE, 2015: 338-345.

[20] SCHWARZ M, RODEHUTSKORS T, SCHREIBER M, et al. Hybrid driving-stepping locomotion with the wheeled-legged robot Momaro[C]// 2016 IEEE International Conference on Robotics and Automation (ICRA), 16-21 May 2016, Stockholm, Sweden. IEEE, 2016: 5589-5595.

[21] HIROSE M, OGAWA K. Honda humanoid robots development[J]. Philosophical Transactions of the Royal Society A: Mathematical, Physical and Engineering Sciences, 2006, 365(1850): 11-19.

[22] FENG S, WHITMAN E, XINJILEFU $X$, et al. Optimization based full body control for the atlas robot[C]// 2014 IEEE-RAS International Conference on Humanoid Robots, 18-20 Nov. 2014, Madrid, Spain. IEEE, 2014: 120-127. 
[23] STENTZ A, HERMAN H, KELLY A, et al. CHIMP, the CMU highly intelligeng mobile platform[J]. Journal of Field Robotics, 2015，32(2): 209-228.

[24] HONG S, LEE Y, PARK K H, et al. Dynamics based motion optimization and operational space control with an experimental rescue robot, HUBO T-100[C]// 2015 IEEE International Conference on Advanced Intelligent Mechatronics (AIM), 7-11 July 2015, Busan, South Korea. IEEE, 2015: 773-778.

[25] ASIMOV I. Runaround[J]. Astounding Science Fiction, 1942, 29(1): 94-103.

[26] OBERER S, SCHRAFT R D. Robot-dummy crash tests for robot safety assessment[C]// Robotics and Automation, 2007 IEEE International Conference on, 10-14 April 2007, Roma, Italy. IEEE, 2007: 2934-2939.

[27] HADDADIN S, ALBU-SCHÄFFER A, HIRZINGER G. Safety evaluation of physical human-robot interaction via crash-testing[J]. Robotics: Science and Systems, 2007, 3: 217-224.

[28] HADDADIN S, ALBU-SCHÄFFER A, HIRZINGER G. Requirements for safe robots: Measurements, analysis and new insights $[\mathrm{J}]$. The International Journal of Robotics Research, 2009, 28(11-12): 1507-1527.

[29] HADDADIN S, HADDADIN S, KHOURY A, et al. On making robots understand safety: Embedding injury knowledge into control[J]. The International Journal of Robotics Research，2012，31(13): 1578-1602.

[30] POVSE B, KORITNIK D, BAJD T, et al. Correlation between impact-energy density and pain intensity during robot-man collision[C]// Biomedical Robotics and Biomechatronics(BioRob), 2010 3rd IEEE RAS and EMBS International Conference on, 26-29 Sept. 2010, Tokyo, Japan. IEEE, 2010: 179-183.

[31] FRAUNHOFER I F F. Determination of verified thresholds for safe human-robot collaboration[EB/OL]. Fraunhofer Institute for Factory Operation and Automation IFF [2019-02-20]. http: //iff.fraunhofer.de.

[32] HADDADIN S. Towards safe robots : Approaching Asimov's 1st law[M]. Springer, 2013.

[33] HADDADIN S, ALBU-SCHAFFER A, DE LUCA A, et al. Collision detection and reaction: A contribution to safe physical human-robot interaction[C]// 2008 IEEE/RSJ International Conference on Intelligent Robots and Systems, 22-26 Sept. 2008, Nice, France. IEEE, 2008: 3356-3363.

[34] PARK J J, SONG J B. Collision analysis and evaluation of collision safety for service robots working in human environments[C]// 2009 International Conference on Advanced Robotics, 22-26 June 2009, Munich, Germany.
IEEE，2009: 1-6.

[35] International Organization for Standardization. ISO.10218-1: 2011: Robots and robotic devices-Safety requirements for industrial robots-Part 1: Robots[S]. Geneva, Switzerland: International Organization for Standardization, 2011.

[36] International Organization for Standardization. ISO.10218-2: 2011: Robots and robotic devices-Safety requirements for industrial robots - Part 2: Robot systems and integration[S]. Geneva, Switzerland: International Organization for Standardization, 2011.

[37] International Organization for Standardization. ISO.13482-2014 Robots and robotic devices - Safety requirements for personal care robots[S]. Geneva, Switzerland : International Organization for Standardization, 2014.

[38] International Organization for Standardization. ISO.ISO/TS 15066 Robots and robotic devices-Collaborative robots[S]. International Organization for Standardization, 2016.

[39] American National Standards Institute. ANSI/RIA R15.06-1999: American National Standard for Industrial Robots and Robot Systems, Safety Requirements[S]. Robotic Industries Association, 1999.

[40] Robotic Industries Association. ANSI/RIA R15.06: 2012 Safety Requirements for industrial robots and robot systems[S]. Ann Arbor: Robotic Industries Association, 2012.

[41] VILLARONGA E F. New Trends in Medical and Service Robots[M]. Springer, 2016.

[42] HADDADIN S, DE LUCA A, ALBU-SCHÄFFER A. Robot collisions: A survey on detection, isolation, and identification[J]. IEEE Transactions on Robotics, 2017, 33(6): 1292-1312.

[43] MI Z, YUAN C M, MA X, et al. Tool orientation optimization for 5-axis machining with $\mathrm{C}$-space method $[\mathrm{J}]$. The International Journal of Advanced Manufacturing Technology, 2017, 88(5-8): 1243-1255.

[44] LACEVIC B， OSMANKOVIC D，ADEMOVIC A. Burs of free c-space: A novel structure for path planning[C]// 2016 IEEE International Conference on Robotics and Automation (ICRA), IEEE, 2016: 70-76.

[45] LÓPEZ-CUSTODIO P C, MÜLLER A, RICO J M, et al. A synthesis method for 1-DOF mechanisms with a cusp in the configuration space[J]. Mechanism and Machine Theory, 2019, 132: 154-175.

[46] YANG X, YANG W, ZHANG H, et al. A new method for robot path planning based artificial potential field[C]// 2016 IEEE 11th Conference on Industrial Electronics and 
Applications (ICIEA), 5-7 June 2016, Hefei, China. IEEE, 2016: 1294-1299.

[47] DE LUCA A, FLACCO F. Integrated control for pHRI: Collision avoidance, detection, reaction and collaboration[C]// Biomedical Robotics and Biomechatronics(BioRob), 2012 4th IEEE RAS\&EMBS International Conference on. IEEE， 2012: 288-295.

[48] ZHANG N, ZHANG Y, MA C, et al. Path planning of six-DOF serial robots based on improved artificial potential field method[C]// 2017 IEEE International Conference on Robotics and Biomimetics (ROBIO). 5-8 Dec. 2017, Macau, China. IEEE, 2017: 617-621.

[49] POLVERINI M P, ZANCHETTIN A M, ROCCO P. Real-time collision avoidance in human-robot interaction based on kinetostatic safety field[C]// 2014 IEEE/RSJ International Conference on Intelligent Robots and Systems, 14-18 Sept. 2014, Chicago, IL, USA. IEEE, 2014: 4136-4141.

[50] LASOTA P A, ROSSANO G F, SHAH J A. Toward safe close-proximity human-robot interaction with standard industrial robots[C]// 2014 IEEE International Conference on Automation Science and Engineering (CASE), 18-22 Aug. 2014, Taipei, Taiwan, China. IEEE, 2014: 339-344.

[51] RYBSKI P, ANDERSON-SPRECHER P, HUBER D, et al. Sensor fusion for human safety in industrial workcells[C]// 2012 IEEE/RSJ International Conference on Intelligent Robots and Systems, 7-12 Oct. 2012, Vilamoura, Portugal. IEEE, 2012: 3612-3619.

[52] FLACCO F, KRÖGER T, DE LUCA A, et al. A depth space approach to human-robot collision avoidance[C]// 2012 IEEE International Conference on Robotics and Automation, 14-18 May 2012, Saint Paul, MN, USA. IEEE, 2012: 338-345.

[53] AZORIN-LÓPEZ J , SAVAL-CALVO M , FUSTER-GUILLÓ A, et al. A predictive model for recognizing human behaviour based on trajectory representation[C]// 2014 International Joint Conference on Neural Networks (IJCNN), 6-11 July 2014, Beijing, China. IEEE, 2014: 1494-1501.

[54] AVANZINI G B, CERIANI N M, ZANCHETTIN A M, et al. Safety control of industrial robots based on a distributed distance sensor[J]. IEEE Transactions on Control Systems Technology, 2014, 22(6): 2127-2140.

[55] VOGEL C, WALTER C, ELKMANN N. A projection-based sensor system for safe physical human-robot collaboration[C]// 2013 IEEE/RSJ International Conference on Intelligent Robots and Systems, 3-7 Nov. 2013, Tokyo, Japan. IEEE, 2013: 5359-5364.
[56] LASOTA P A, SHAH J A. Analyzing the effects of human-aware motion planning on close-proximity human-robot collaboration[J]. Human factors, 2015, 57(1): 21-33.

[57] SPONG M W, HUTCHINSON S A, VIDYASAGAR M. Robot modeling and control[J]. IEEE Control Systems, 2006, 26(6): 113-115.

[58] OZGOLI S, TAGHIRAD H D. A survey on the control of flexible joint robots[J]. Asian Journal of Control, 2006, 8(4): 332-344.

[59] KHAN Z A, CHACKO V, NAZIR H. A review of friction models in interacting joints for durability design[J]. Friction, 2017, 5(1): 1-22.

[60] FLACCO F, DE LUCA A. Residual-based stiffness estimation in robots with flexible transmissions[C]// 2011 IEEE International Conference on Robotics and Automation. 9-13 May 2011, Shanghai, China. IEEE, 2011: 5541-5547.

[61] FLACCO F, DE LUCA A. Stiffness estimation and nonlinear control of robots with variable stiffness actuation[J]. IFAC Proceedings Volumes, 2011, 44(1): 6872-6879.

[62] MENARD T, GRIOLI G, BICCHI A. A real time robust observer for an Agonist-Antagonist Variable Stiffness Actuator[C]// 2013 IEEE International Conference on Robotics and Automation, 6-10 May 2013, Karlsruhe, Germany. IEEE, 2013: 3988-3993.

[63] MÉNARD T, GRIOLI G, BICCHI A. A stiffness estimator for agonistic-antagonistic variable-stiffnessactuator devices[J]. IEEE Transactions on Robotics, 2014, 30(5): 1269-1278.

[64] JIA J, ZHANG M, ZANG X, et al. Dynamic parameter identification for a manipulator with joint torque sensors based on an improved experimental design[J]. Sensors, 2019, 19(10): 2248

[65] JIN H , LIU Z , ZHANG H , et al. A dynamic parameter identification method for flexible joints based on adaptive control[J]. IEEE/ASME Transactions on Mechatronics, 2018, 23(6): 2896-2908.

[66] LICHIARDOPOL S, VAN DE WOUW N, NIJMEIJER H. Robust disturbance estimation for human-robotic comanipulation[J]. International Journal of Robust and Nonlinear Control, 2014, 24(12): 1772-1796.

[67] WAHRBURG A, ROBERTSSON A, MATTHIAS B, et al. Improving contact force estimation accuracy by optimal redundancy resolution[C]// 2016 IEEE/RSJ International Conference on Intelligent Robots and Systems (IROS), 9-14 Oct. 2016, Daejeon, South Korea. IEEE, 2016: 3735-3741. 
[68] WAHRBURG A, MORARA E, CESARI G, et al. Cartesian contact force estimation for robotic manipulators using Kalman filters and the generalized momentum[C]// 2015 IEEE International Conference on Automation Science and Engineering (CASE), 24-28 Aug. 2015, Gothenburg, Sweden. IEEE, 2015: 1230-1235.

[69] WAHRBURG A, BÖS J, LISTMANN K D, et al. Motor-current-based estimation of cartesian contact forces and torques for robotic manipulators and its application to force control[J]. IEEE Transactions on Automation Science and Engineering, 2017, 15(2): 879-886.

[70] HU J, XIONG R. Contact force estimation for robot manipulator using semiparametric model and disturbance Kalman filter[J]. IEEE Transactions on Industrial Electronics, 2018, 65(4): 3365-3375.

[71] MAKAROV M, CALDAS A, GROSSARD $\mathrm{M}$, et al. Adaptive filtering for robust proprioceptive robot impact detection under model uncertainties[J]. IEEE/ASME Transactions on Mechatronics, 2014， 19(6): 1917-1928.

[72] BRIQUET-KERESTEDJIAN $\mathrm{N}$, MAKAROV $\mathrm{M}$, GROSSARD $\mathrm{M}$, et al. Generalized momentum based-observer for robot impact detection-Insights and guidelines under characterized uncertainties[C]// 2017 IEEE Conference on Control Technology and Applications (CCTA), 27-30 Aug. 2017, Mauna Lani, HI, USA. IEEE, 2017: 1282-1287.

[73] SHARKAWY A N, ASPRAGATHOS N. Human-robot collision detection based on neural networks[J]. Int. J. Mech. Eng. Robot. Res., 2018, 7(2): 150-157.

[74] INDRI M, TRAPANI S, LAZZERO I. A general procedure for collision detection between an industrial robot and the environment[C]// 2015 IEEE 20th Conference on Emerging Technologies \& Factory Automation, 8-11 Sept. 2015, Luxembourg, Luxembourg. IEEE, 2015: 1-8.

[75] INDRI M, TRAPANI S, LAZZERO I. Development of a virtual collision sensor for industrial robots[J]. Sensors, 2017, 17(5): 1148 .

[76] SOTOUDEHNEJAD V, TAKHMAR A, KERMANI M $\mathrm{R}$, et al. Counteracting modeling errors for sensitive observer-based manipulator collision detection[C]// 2012 IEEE/RSJ International Conference on Intelligent Robots and Systems, 7-12 Oct. 2012, Vilamoura, Portugal. IEEE, 2012: 4315-4320.

[77] SOTOUDEHNEJAD V, KERMANI M R. Velocity-based variable thresholds for improving collision detection in manipulators[C]// 2014 IEEE International Conference on Robotics and Automation (ICRA), 31 May-7 June 2014, Hong Kong, China. IEEE, 2014: 3364-3369.
[78] JUNG B, CHOI H R, KOO J C, et al. Collision detection using band designed disturbance observer[C]// 2012 IEEE International Conference on Automation Science and Engineering (CASE), 20-24 Aug. 2012, Seoul, South Korea. IEEE， 2012: 1080-1085.

[79] JUNG B, KOO J C, CHOI H R, et al. Human-robot collision detection under modeling uncertainty using frequency boundary of manipulator dynamics[J]. J. Mech. Sci. Tech., 2014, 28(11): 4389-4395.

[80] HO C N, SONG J B. Collision detection algorithm robust to model uncertainty[J]. International Journal of Control, Automation and Systems, 2013, 11(4): 776-781.

[81] ERDEN M S, TOMIYAMA T. Human-intent detection and physically interactive control of a robot without force sensors[J]. IEEE Transactions on Robotics, 2010, 26(2): 370-382.

[82] CHO C N, KIM J H, KIM Y L, et al.Collision detection algorithm to distinguish between intended contact and unexpected collision[J]. Advanced Robotics, 2012, 26(16): 1825-1840.

[83] GERAVAND M, FLACCO F, DE LUCA A. Human-robot physical interaction and collaboration using an industrial robot with a closed control architecture[C]// 2013 IEEE International Conference on Robotics and Automation, 6-10 May 2013, Karlsruhe, Germany. IEEE, 2013: 4000-4007.

[84] LI Y, GE S S. Human-robot collaboration based on motion intention estimation[J]. IEEE/ASME Transactions on Mechatronics, 2014, 19(3): 1007-1014.

[85] GOLZ S, OSENDORFER C, HADDADIN S. Using tactile sensation for learning contact knowledge : Discriminate collision from physical interaction[C]// 2015 IEEE International Conference on Robotics and Automation (ICRA), 26-30 May 2015, Seattle, WA, USA. IEEE, 2015: 3788-3794.

[86] PARK D I, KIM H, PARK C, et al. Automatic assembly method with the passive compliant device[C]// 2017 11th Asian Control Conference (ASCC), 17-20 Dec. 2017, Gold Coast, QLD, Australia. IEEE, 2017: 347-348.

[87] HUANG T H, HUANG H P, KUAN J Y. Mechanism and control of continuous-state coupled elastic actuation[J]. J. Intell. Robot Syst., 2014, 74(3-4): 571-587.

[88] MIURA K, KATSURA S. Experimental validation of forward-and back-drivable characteristics on series clutch actuators using acceleration control[C]// 2016 IEEE International Conference on Advanced Intelligent Mechatronics (AIM), 12-15 July 2016, Banff, AB, Canada. IEEE, 2016: 716-720.

[89] FICUCIELLO F, VILLANI L, SICILIANO B. Variable 
impedance control of redundant manipulators for intuitive human-robot physical interaction[J]. IEEE Transactions on Robotics, 2015, 31(4): 850-863.

[90] LI X, PAN Y, CHEN G, et al. Adaptive human-robot interaction control for robots driven by series elastic actuators[J]. IEEE Transactions on Robotics, 2017, 33(1): 169-182.

[91] JEONG H, CHEONG J, KWON S J. Dual-mode variable stiffness actuator using two-stage worm gear transmission for safe robotic manipulators[J]. International Journal of Precision Engineering and Manufacturing, 2015, 16(8): 1761-1769.

[92] KAMADAN A, KIZILTAS G, PATOGLU V. Co-design strategies for optimal variable stiffness actuation[J]. IEEE/ASME Transactions on Mechatronics, 2017, 22(6): 2768-2779.

[93] GREBENSTEIN M, CHALON M, FRIEDL W, et al. The hand of the DLR hand arm system: Designed for interaction[J]. International Journal of Robotics Research, 2012, 31(13): 1531-1555.

[94] RAIBERT M H, CRAIG J J. Hybrid position/force control of manipulators[J]. Journal of Dynamic Systems, Measurement, and Control, 1981, 103(2): 126-133.

[95] HOGAN N. Impedance control : An approach to manipulation: Part II-Implementation[J]. Journal of Dynamic Systems, Measurement, and Control, 1985, 107(1): 8-16.

[96] SENTIS L, KHATIB O. Prioritized multi-objective dynamics and control of robots in human environments[C]// 4th IEEE/RAS International Conference on Humanoid Robots, 2004, 10-12 Nov. 2004, Santa Monica, CA, USA, USA. IEEE, 2004: 764-780.

[97] ALBU-SCHAFFER A , HIRZINGER G. Cartesian impedance control techniques for torque controlled light-weight robots[C]// Proceedings 2002 IEEE International Conference on Robotics and Automation, 11-15 May 2002, Washington, DC, USA. IEEE, 2002, 1: 657-663.

[98] PLATT R , ABDALLAH M , WAMPLER C. Multiple-priority impedance control[C]// 2011 IEEE International Conference on Robotics and Automation, 9-13 May 2011, Shanghai, China. IEEE, 2011: 6033-6038.

[99] DE LUCA A, FERRAJOLI L. Exploiting robot redundancy in collision detection and reaction[C]// 2008 IEEE/RSJ International Conference on Intelligent Robots and Systems, 22-26 Sept. 2008, Nice, France. IEEE, 2008: 3299-3305.

[100] SADEGHIAN H, KESHMIRI M, VILLANI L, et al. Null-space impedance control with disturbance
observer[C]// 2012 IEEE/RSJ International Conference on Intelligent Robots and Systems, 7-12 Oct. 2012, Vilamoura, Portugal. IEEE, 2012: 2795-2800.

[101] SADEGHIAN H, VILLANI L, KESHMIRI $M$, et al.Task-space control of robot manipulators with null-space compliance[J]. IEEE Transactions on Robotics, 2014, 30(2): 493-506.

[102] RYOO M S. Human activity prediction : Early recognition of ongoing activities from streaming videos[C]// 2011 International Conference on Computer Vision, 6-13 Nov. 2011, Barcelona, Spain. IEEE, 2011: 1036-1043.

[103] RYOO M S, FUCHS T J, XIA L, et al. Robot-centric activity prediction from first-person videos: What will they do to me?[C]// 2015 10th ACM/IEEE International Conference on Human-Robot Interaction (HRI), 2-5 March 2015, Portland, OR, USA. IEEE, 2015: 295-302.

[104] KOPPULA H S, SAXENA A. Anticipating human activities using object affordances for reactive robotic response[J]. IEEE Transactions on Pattern Analysis and Machine Intelligence, 2016，38(1): 14-29.

[105] MAINPRICE J , BERENSON D. Human-robot collaborative manipulation planning using early prediction of human motion[C]// 2013 IEEE/RSJ International Conference on Intelligent Robots and Systems, 3-7 Nov. 2013, Tokyo, Japan. IEEE, 2013: 299-306.

[106] PÉREZ-D'ARPINO C, SHAH J A. Fast target prediction of human reaching motion for cooperative human-robot manipulation tasks using time series classification[C]// 2015 IEEE International Conference on Robotics and Automation (ICRA), 26-30 May 2015, Seattle, WA, USA. IEEE, 2015: 6175-6182.

[107] DOMINEY P F, METTA G, NORI F, et al. Anticipation and initiative in human-humanoid interaction[C]// Humanoids 2008-8th IEEE-RAS International Conference on Humanoid Robots, 1-3 Dec. 2008, Daejeon, South Korea. IEEE, 2008: 693-699.

[108] NIKOLAIDIS S, LASOTA P, ROSSANO G, et al. Human-robot collaboration in manufacturing : Quantitative evaluation of predictable, convergent joint action[C]// IEEE ISR 2013, 24-26 Oct. 2013, Seoul, South Korea. IEEE， 2013: 1-6.

[109] NIKOLAIDIS S, RAMAKRISHNAN R, GU K, et al.Efficient model learning from joint-action demonstrations for human-robot collaborative tasks[C]// Proceedings of the 10th Annual ACM/IEEE International Conference on Human-robot Interaction, 2-5 March 2015, Portland, OR, USA. IEEE, 2015: 189-196. 
[110] LI K, FU Y. Prediction of human activity by discovering temporal sequence patterns[J]. IEEE T. Pattern Anal., 2014, 36(8): 1644-1657.

[111] JIANG Y, SAXENA A. Modeling high-dimensional humans for activity anticipation using Gaussian process latent CRFs[C]// Robotics: Science and Systems, 2014: $1-8$.

[112] MAINPRICE J, HAYNE R, BERENSON D. Predicting human reaching motion in collaborative tasks using inverse optimal control and iterative re-planning[C]// 2015 IEEE International Conference on Robotics and Automation (ICRA), 26-30 May 2015, Seattle, WA, USA. IEEE， 2015: 885-892.

[113] TAKANO W, IMAGAWA H, NAKAMURA Y. Prediction of human behaviors in the future through symbolic inference[C]// 2011 IEEE International Conference on Robotics and Automation, 9-13 May 2011, Shanghai, China. IEEE, 2011: 1970-1975.

[114] XIAO S, WANG Z, FOLKESSON J. Unsupervised robot learning to predict person motion[C]// 2015 IEEE International Conference on Robotics and Automation (ICRA), 26-30 May 2015, Seattle, WA, USA. IEEE, 2015: 691-696.

[115] KUDERER M, KRETZSCHMAR H, SPRUNK C, et al. Feature-based prediction of trajectories for socially compliant navigation[C]// Robotics : Science and Systems, MIT Press, 2012: 504.

[116] WALKER J, GUPTA A, HEBERT M. Patch to the future: Unsupervised visual prediction[C]// Proceedings of the IEEE Conference on Computer Vision and Pattern Recognition, 23-28 June 2014, Columbus, OH, USA. 2014: 3302-3309.

[117] GOODFELLOW I, POUGET-ABADIE J, MIRZA M, et al. Generative adversarial nets $[\mathrm{C}] / /$ Advances in Neural Information Processing Systems, 2014 : 2672-2680

[118] MAO X, LI Q, XIE H, et al. Least squares generative adversarial networks $[\mathrm{C}] / /$ Proceedings of the IEEE International Conference on Computer Vision, 22-29 Oct. 2017, Venice, Italy. IEEE, 2017: 2794-2802.

[119] CRESWELL A, WHITE T, DUMOULIN V, et al. Generative adversarial networks: An overview[J]. IEEE Signal Processing Magazine, 2018, 35(1): 53-65.

[120] 戴建生. 机构学与机器人学的几何基础与旋量代数 [M]. 北京: 高等教育出版社, 2014.

DAI Jiansheng. Geometrical foundation $\mathrm{s}$ and screw algebra for mechanisms and robotics[M]. Beijing: Higher Education Press, 2014.

[121] WEI Y, XU Q. An overview of micro-force sensing techniques[J]. Sensors and Actuators A: Physical, 2015, 234: $359-374$.

作者简介: 贾计东, 男, 1992 年出生, 博士研究生。主要研究方向为人 机共融技术。

mail: jiajidong.0724@163.com

张明路(通信作者), 男, 1964 年出生, 博士, 教授, 博士研究生导师。 主要研究方向为机器人技术, 智能主体技术及应用。

E-mail: zhangml@hebut.edu.cn 